Obstetrics

\title{
Factors associated with maternal mortality among patients meeting criteria of severe maternal morbidity and near miss
}

\author{
Hesly M.P. Lima ${ }^{1 *}$ | Francisco Herlânio C. Carvalho ${ }^{2}$ | Francisco Edson L. Feitosa ${ }^{3}$ | \\ George C. Nunes ${ }^{3}$
}

${ }^{1}$ Department of Community Health, Federal University of Ceará, Health Sciences Center, Fortaleza, Ceará, Brazil

${ }^{2}$ Department of Maternal and Child, Federal University of Ceará, Health Sciences Center, Fortaleza, Ceará, Brazil

${ }^{3}$ Maternity School, Assis Chateaubriand, Fortaleza, Ceará, Brazil

${ }^{*}$ Correspondence

Hesly M.P. Lima, Department of Community Health, Fortaleza, CE, Brazil.

Email: heslymartinsplima@hotmail.com

\begin{abstract}
Objective: To evaluate factors associated with maternal death among women experiencing life-threatening conditions during pregnancy, childbirth, or within 42 days of termination of pregnancy.

Methods: A secondary analysis of data prospectively collected in a Brazilian multicenter cross-sectional study between July 2009 and June 2010 was conducted. Women were identified who delivered at a hospital in Ceará and who had potentially life-threatening conditions. Stepwise logistic regression was used to identify factors associated with maternal death.

Results: Overall, 941 women were identified and 11 died. Among criteria for severe maternal morbidity, eclampsia (adjusted odds ratio [aOR] 203.70, 95\% Cl 5.03 to 8254.20; $P=0.005$ ) and intensive care unit (ICU) admission (aOR 69.30, 95\% Cl 6.63724.26; $P<0.001)$ were risk factors for progression to death, whereas use of magnesium sulfate (aOR $0.002,95 \% \mathrm{Cl}<0.01-0.11 ; P=0.002$ ) was a protective factor. Meeting near-miss criteria other than survival (aOR 5.96, 95\% Cl 1.69-20.98; $P=0.005$ ) was associated with maternal death. Of criteria for near miss, management criteria were most strongly associated with maternal death: all 11 women who died met some management criteria.

Conclusion: Among WHO's criteria for severe maternal morbidity and near miss, eclampsia, low oxygen saturation, ICU admission, intubation, mechanical ventilation, and cardiopulmonary resuscitation were most associated with maternal death. Use of magnesium sulfate was a protective factor.
\end{abstract}

\section{KEYWORDS}

Cause of death; Maternal morbidity; Maternal mortality; Morbidity; Near miss approach to maternal health; $\mathrm{WHO}$

\section{1 | INTRODUCTION}

In 2000, a set of targets including infant and maternal indicators were established by WHO as part of the Millennium Development Goals. ${ }^{1}$ For maternal health, the objective was to reduce the maternal mortality ratio by three-quarters of the 1990 value by $2015 .^{2}$
Maternal mortality is one of the most important indicators of global inequality in health. The statistics reflect deficits in access to, and quality of, health services, and the failure of public policies to promote women's health. ${ }^{3}$ To reduce maternal mortality, it is necessary to determine the causes of death and acquire a better understanding of the unmet needs and deficiencies that lead to poor obstetric outcomes. In 2013, there were 1686 maternal deaths in 
Brazil, 617 of which were in the Northeast Region and 93 in the state of Ceará alone. ${ }^{4}$

Formulating plans and actions on the basis of only evaluations of maternal death is not enough. Cases of severe maternal morbidityserious complications of pregnancy, such as hemorrhagic disordersand near miss-survival of a life-threatening complication occurring during pregnancy, childbirth, or within 42 days of termination of pregnancy-can inform strategies for epidemiologic investigation that are more comprehensive and informative than those based on cases of death only. Therefore, WHO has standardized its diagnostic criteria for severe maternal morbidity and near miss. ${ }^{5-7}$ The division of maternal near-miss criteria into three groups-clinical, laboratory, and management-has been shown to facilitate the identification of severe cases, even when laboratory data or other techniques are not available. ${ }^{7}$ WHO has also produced a list of potentially life-threatening conditions ${ }^{7}$-including hemorrhagic disorders, other systemic disorders, hypertensive disorders, and severe management indicators-that could be used prospectively to identify women who could go on to die or to become a case of near miss.

Studies of maternal near miss can meet the emerging need to determine the size and characteristics of this population relative to the unfavorable outcome of maternal death. The aim of the present study was therefore to evaluate the factors associated with maternal death among women experiencing life-threatening conditions during pregnancy, childbirth, or within 42 days of termination of pregnancy who were treated in a tertiary referral center for maternal and neonatal health.

\section{2 | MATERIALS AND METHODS}

The present study was a secondary analysis of data prospectively collected from medical records in a multicenter study by The Brazilian Network for Surveillance of Severe Maternal Morbidity Group between July 1 2009, and 30 June, 2010. It was based on a subset of data from women who were treated at the MEAC (Assis Chateaubriand Maternity Teaching Hospital), Federal University of Ceará, in Fortaleza, Brazil. The present study was approved by the Ethics Committee for Research at MEAC in accordance with the principles that regulate research on human beings (protocol number 958,601). Informed consent was not deemed necessary because the study data were obtained from medical records without identification of the women.

The parent cross-sectional study was implemented in 27 hospitals across all Brazilian regions. Data relating to the WHO criteria for near miss and potentially life-threatening conditions ${ }^{7}$ were collected from medical records. Patients with any of the potentially life-threatening conditions defined by $\mathrm{WHO}$, those meeting near-miss criteria, those who died, and those who were referred to another health facility were included. The parent study's aim was to identify cases of severe maternal morbidity during pregnancy, delivery, and the postpartum period in Brazil to determine whether women at risk of a worse outcome can be identified by application of the WHO criteria.
For the present analysis, data for women treated at MEAC only were included. The variable outcome was maternal death, and the exposure variables were criteria for severe maternal morbidity and the WHO criteria for life-threatening conditions (i.e. criteria other than survival used to identify near-miss cases). Eight criteria were used to define severe maternal morbidity; seven of these were from the WHO proposed list of potentially life-threatening conditions. ${ }^{7}$ Overall, the criteria used to define maternal morbidity were severe pre-eclampsia, eclampsia, HELLP syndrome (hemolysis, elevated liver enzymes, low platelet count), severe sepsis, hemorrhagic disorders, admission to intensive care unit (ICU), blood transfusion, and use of magnesium sulfate owing to its wide use in the study location and its association with severe hypertensive conditions. The following conditions were considered as a group of hemorrhagic syndromes: abruptio placentae, placenta previa, uterine rupture, tubal pregnancy, and coagulopathy. For the purpose of data analysis, the criteria were considered, not the classification, because women could meet more than one criterion of severe maternal morbidity or near miss.

The data were analyzed using Stata version 11.2 (StataCorp, College Station, TX, USA). The exposure variables were characterized as dichotomous (yes/no). Continuous variables were reported as mean $\pm S D$. Differences were evaluated by $\chi^{2}$ or Fisher association test as applicable. First, the variables that were significant at a $P$ value of less than 0.05 were examined in a bivariate analysis to calculate the crude odds ratios (ORs) for association with maternal death. The variables that presented a confidence interval $(\mathrm{Cl})$ that did not exceed 1 , and had a $P$ value of less than 0.05 in the bivariate analysis were included in a stepwise multiple logistic regression to calculate the adjusted odds ratio (aOR) for association with maternal death.

\section{3 | RESULTS}

In the parent study, there were 9555 identified cases of severe maternal morbidity, 770 cases of maternal near miss, and 140 deaths. Hypertensive disease was the leading cause of complications. ${ }^{8}$ In the present analysis of data collected at MEAC, 941 women were identified: 50 had maternal near miss, 880 had severe maternal morbidity, and 11 died. During the study period, there were 4617 live births at the study institution, yielding a maternal near-miss rate of 10.8 per 1000 live births, a severe maternal morbidity rate of 190.6 per 1000 live births, and a maternal mortality ratio of 238.2 per 100000 live births.

The mean maternal age was $25 \pm 7.52$ years (range $12-46$ ); 592 (62.9\%) women were aged 20-34 years. Overall, 729 (77.5\%) women stated that they did not have a partner. Among 776 women for whom schooling data were available, 482 (62.1\%) reported that they had more than 8 years schooling and 9 (1.2\%) were illiterate. Mean number of previous pregnancies was $2 \pm 1$. Regarding parturition, 506 (53.8\%) of the women were nulliparous. In total, 716 (76.1\%) women had not had a prior spontaneous abortion. At least one previous cesarean delivery was reported for 151 (16.0\%) women, and 64 (6.8\%) women 
had undergone two or more cesareans. Regarding prenatal care, 622 (66.1\%) attended fewer than six appointments.

Among the 880 women with severe maternal morbidity, 559 (63.5\%) were aged 20-34 years. More than 8 years of education was reported for 466 (53.0\%). Overall, 679 (77.2\%) had no partner, 478 (54.3\%) were nulliparous, 207 (23.5\%) had a previous spontaneous abortion, 143 (16.3\%) had previously undergone at least one cesarean delivery, and 568 (64.5\%) attended fewer than six prenatal appointments. In relation to pre-existing conditions, 94 (10.7\%) had chronic hypertension in a previous pregnancy and 23 (2.6\%) had diabetes mellitus before pregnancy.

Among the 61 women with life-threatening conditions (50 nearmiss cases and 11 deaths), 33 (54.1\%) were aged 20-34 years. Fewer than 8 years of education was reported for 12 (19.7\%). Overall, 50 (82.0\%) had no partner, 33 (54.1\%) were nulliparous, 43 (70.5\%) had no previous spontaneous abortion, 6 (9.8\%) had previously undergone two or more cesarean deliveries, and 54 (88.5\%) had attended fewer than six prenatal appointments. Six (9.8\%) women had chronic hypertension and 2 (3.3\%) had diabetes mellitus before pregnancy.

Neonatal death was reported for 7 (1.1\%) of 622 newborns. Overall, 443 (53.8\%) of 823 neonates were male, and 269 (34.1\%) of 788 weighed less than $2499 \mathrm{~g}$ (mean $2741 \pm 827 \mathrm{~g}$, range $405-$ $5040)$. In relation to gestational age, $479(60.7 \%)$ of 789 were born at more than 37 weeks (range 6-42, mean 36.1 \pm 4.1 ). Regarding vitality, 650 (82.5\%) and 764 (97.0\%) of 788 had an Apgar score of more than 7 at 1 and 5 minutes, respectively. Cesarean delivery was more prevalent than vaginal delivery $(683 / 873$ [78.2\%] vs $171 / 873$ [19.6\%]).

Among the 11 cases of maternal death, 10 involved admission to the ICU, 7 the use of blood products, 5 hypertensive syndromes (severe pre-eclampsia, eclampsia, or HELLP syndrome), 4 hemorrhagic disorders (abruptio placentae, placenta previa, uterine rupture, tubal pregnancy, or coagulopathy), and 4 severe sepsis.

Table 1 shows the data of the bivariate analysis and logistic regression for maternal death based on the criteria for severe maternal morbidity among the 941 study women. Except for HELLP syndrome, for which the 82 affected women survived, all other criteria showed an association with maternal death. After calculating the crude OR, these criteria continued to show a significant association with maternal death (Table 1).

After stepwise logistic analysis, the following variables remained in the final model: severe pre-eclampsia, eclampsia, hemorrhagic disorders, magnesium sulfate, and admittance to ICU. Notably magnesium sulfate was the only criterion that acted as a protective factor after the final analysis (aOR $0.002,95 \% \mathrm{Cl}<0.001$ to $0.11 ; P=0.002$ ) (Table 1).

Among the 61 women with life-threatening conditions, 26 met only one diagnostic criterion and none of these women died. Among 17 women who met four or more of the 25 criteria, 8 (47.1\%) died. Meeting criteria of near miss was found to be significantly associated with maternal death ( $P=0.002)$ (Table 2$)$.

The classification of near miss by WHO is grouped into clinical, laboratory, and management criteria. Among women with life-threatening conditions, 9 (28.1\%) of 32 meeting clinical near-miss criteria, 5 (15.6\%) of 32 meeting laboratory near-miss criteria, and 11 (26.8\%) of 41 meeting management criteria died.

Among the clinical criteria, the most prevalent were loss of consciousness for 12 hours or more (9/32 [28.1\%]), respiratory frequency above 40 or below 6 incursions per minute (8/32 [25.0\%]), loss of consciousness and absence of pulse (7/32 [21.9\%]), and shock (7/32 [21.9\%]). Of the laboratory criteria, acute thrombocytopenia (20/32 [62.5\%]) was the most prevalent specific criterion. Continuous use of vasoactive drugs (24/41 [58.5\%]) and intubation and ventilation not associated with anesthesia for more than 1 hour (23/41 [56.1\%]) were the most prevalent management criteria.

Among the clinical criteria, coagulation disorders were observed in two cases of near miss, which both resulted in maternal death; acute cyanosis and non-controlled convulsions were associated with death for half the women affected ( $1 / 2$ and $2 / 4$, respectively). Among the laboratory criteria, both women with a $\mathrm{pH}$ of less than 7.1 died, whereas four of eight cases of oxygen saturation lower than $90 \%$ for longer than 1 hour resulted in maternal death. Dialysis for acute renal failure and cardiopulmonary resuscitation led to death in three of five cases, and five of ten cases, respectively. Among the 41 patients meeting management criteria for near miss. $11(26.8 \%)$ died.

\section{4 | DISCUSSION}

During study period, there were 880 cases of severe maternal morbidity and 61 cases of life-threatening maternal conditions at MEAC. Among the diagnostic criteria for severe maternal morbidity, eclampsia and need for ICU admission were identified as risk factors for progression to death, whereas use of magnesium sulfate acted as a protective factor. Regarding life-threatening conditions, maternal death was associated with having any of WHO's near-miss clinical criteria, an oxygen saturation of less than $90 \%$ for more than 1 hour among the laboratory criteria, and need for intubation and ventilation for a period of 1 hour or more (not related to anesthesia) and need for cardiopulmonary resuscitation among the management criteria.

Among the determinant criteria for near miss, the most strongly associated with maternal death was the presence of management criteria: all 11 cases of life-threatening conditions that ended in maternal death presented with at least one management criterion. This demonstrates that this group of $\mathrm{WHO}$ criteria identifies women requiring the greatest attention and caution during health care.

Notably, none of the demographic characteristics of the patients showed any association with maternal death in the present study, unlike the clinical situation at hospital admission and the presence of severe maternal morbidity and maternal near-miss criteria. An increased incidence of severe maternal morbidity and near miss among women aged 20-34 years has been found in other studies, ${ }^{5,9-11}$ suggesting the need for close care of women in this age group. 
TAB LE 1 Bivariate analysis and logistic regression of conditions of severe maternal morbidity associated with maternal death ( $\mathrm{n=941).}$

\begin{tabular}{|c|c|c|c|c|c|c|c|}
\hline $\begin{array}{l}\text { Factors for severe } \\
\text { maternal morbidity }\end{array}$ & Died $^{a}$ & Survived $^{a}$ & $P$ value $^{b}$ & \multicolumn{2}{|l|}{ Bivariate analysis } & \multicolumn{2}{|c|}{ Multiple logistic regression } \\
\hline Severe pre-eclampsia & & & $<0.001$ & $0.05(0.01-0.24)$ & $<0.001$ & $31.24(1.25-782.90)$ & 0.036 \\
\hline Yes & $2 / 758(0.3)$ & 756/758 (99.7) & & & & & \\
\hline No & 9/183 (4.9) & $174 / 183(95.1)$ & & & & & \\
\hline No & $8 / 881(0.9)$ & $873 / 881(99.1)$ & & & & & \\
\hline HELLP syndrome ${ }^{c}$ & & & 0.612 & - & - & - & - \\
\hline Yes & $0 / 82$ & $82 / 82(100.0)$ & & & & & \\
\hline No & $11 / 859(1.3)$ & $848 / 859(98.7)$ & & & & & \\
\hline $\begin{array}{l}\text { Hemorrhagic } \\
\text { disorders }\end{array}$ & & & 0.020 & $5.14(1.48-17.90)$ & 0.010 & $4.41(1.02-19.02)$ & 0.047 \\
\hline Yes & $4 / 97(4.1)$ & 93/97 (95.9) & & & & & \\
\hline No & $7 / 844(0.8)$ & $837 / 844(99.2)$ & & & & & \\
\hline Magnesium sulfate & & & $<0.001$ & $0.08(0.02-0.29)$ & $<0.001$ & $0.002(<0.01-0.11)$ & 0.002 \\
\hline Yes & $4 / 815(0.5)$ & $811 / 815(99.5)$ & & & & & \\
\hline No & $7 / 126(5.6)$ & $119 / 126(94.4)$ & & & & & \\
\hline $\begin{array}{l}\text { Admission to the } \\
\text { intensive care unit }\end{array}$ & & & $<0.001$ & $83.00(10.51-655.20)$ & $<0.001$ & $69.30(6.63-724.26)$ & $<0.001$ \\
\hline
\end{tabular}

Abbreviations: OR, odds ratio; $\mathrm{Cl}$, confidence interval; aOR, adjusted odds ratio.

${ }^{a}$ Values are given as number/total number (percentage).

${ }^{\mathrm{b}}$ By Fisher exact test.

${ }^{c}$ Hemolysis, elevated liver enzymes, low platelet count.

Nulliparous women formed the biggest group in the present analysis, which has also been observed in other studies. ${ }^{9,12}$ In the present study, by contrast with other studies, ${ }^{5,10-13}$ women with severe and near-miss maternal morbidity who had no partner or were not married were not the largest groups.

A deficiency in prenatal care, which can lead to complications during pregnancy, is a reality in Brazil and has been reported in other studies. ${ }^{10,13,14}$ In the present study, 568 (64.5\%) of women with severe maternal morbidity and 54 (88.5\%) of women with near miss or death had attended fewer than six prenatal appointments.

Among women with severe maternal morbidity, 143 (16.3\%) had undergone a previous cesarean and, among those with near miss or death, $6(10 \%)$ had two or more previous cesareans. This strengthens the need for studies of the prevalence of this type of delivery in relation to severe maternal morbidity and maternal near miss, especially because the number of cesarean procedures is increasing in Brazil. ${ }^{14-16}$
Hypertensive disorders in pregnancy include pre-eclampsia, eclampsia, and HELLP syndrome, the last of which a leading cause of maternal morbidity and mortality in the USA and globally, accounting for $10 \%-15 \%$ of maternal deaths. ${ }^{17,18}$ In the present study, severe pre-eclampsia and pre-eclampsia were identified as severe maternal morbidity factors associated with the death of these women, as found in other studies. $5,9,11,12,19$

Hemorrhage is also a major cause of maternal death worldwide, accounting for approximately $50 \%$ of deaths in some low- and middleincome countries. ${ }^{20}$ The reason for this high rate of mortality is the lack of any adequate medical care or basic life support, despite the existence of well-defined protocols for prevention. ${ }^{20}$ In the present study, an association between hemorrhagic disorders (abruptio placentae, placenta previa, uterine rupture, tubal pregnancy, and coagulopathy) and maternal death was observed among women with severe maternal morbidity. 
TAB LE 2 Bivariate analysis and logistic regression of near miss criteria other than survival associated with maternal death ( $n=61$ ).

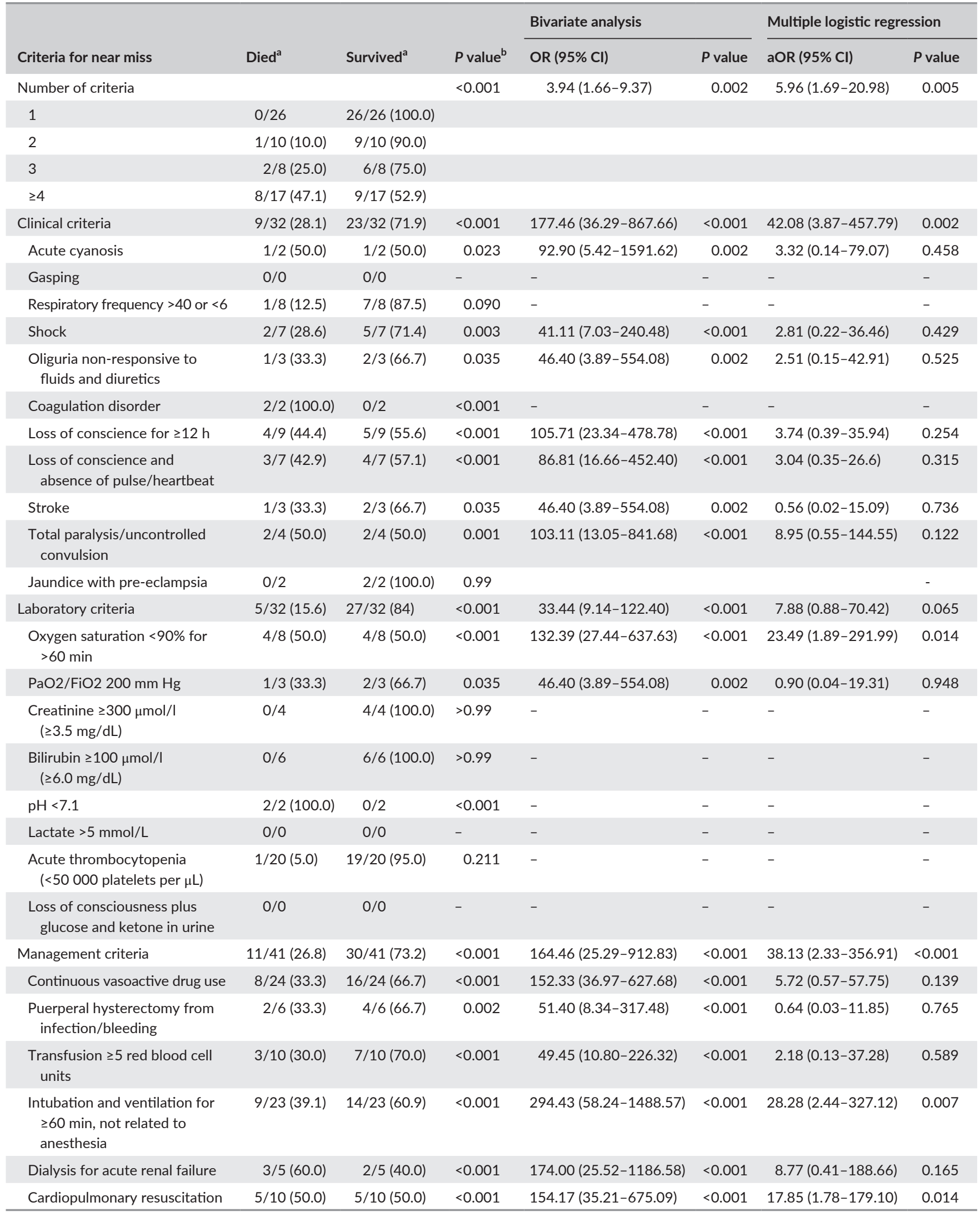

Abbreviations: $\mathrm{OR}$, odds ratio; $\mathrm{Cl}$, confidence interval; aOR, adjusted odds ratio; $\mathrm{Pa}_{2} / \mathrm{FiO}_{2}$, ratio of arterial oxygen partial pressure to fractional inspired oxygen.

${ }^{a}$ Values are given as number/total number (percentage).

${ }^{\mathrm{b}}$ By Pearson $\chi^{2}$ test or Fisher exact test. 
The Brazilian Network for Surveillance of Severe Maternal Morbidity Group study ${ }^{13}$ verified an incidence of hemorrhagic disorders of $8 \%$ (767/9555) before and during labor among women who had obstetric complications. Hemorrhagic disorders before and during labor were also responsible for $7.1 \%(613 / 8645)$ of cases of severe maternal morbidity, $18.2 \%$ (140/770) of cases of maternal near miss, and $10 \%(14 / 140)$ of cases of maternal death. ${ }^{13}$

A study in various countries by Geller et al. ${ }^{5}$ verified the clinical factors associated with women's progression from morbidity to mortality, concluding that hemorrhage was the most common diagnosis among cases of near miss (39.4\%), whereas hypertensive disorders (pre-eclampsia, eclampsia, and HELLP syndrome) were the most prevalent criteria among cases of severe morbidity (47.5\%). Cardiac disorders were reported to be responsible for $21.6 \%$ of the maternal deaths. ${ }^{5}$ Similarly, in the present study, 20 (38.8\%) women with maternal near miss presented with thrombocytopenia, and hemorrhagic disorders were also the most frequent criterion of severe morbidity.

The clinical pathology and obstetric gravity of severe maternal morbidity and maternal near miss support the fact that clinical criteria of near miss, as defined by WHO, were the group of criteria most associated with maternal death because they are easily observed criteria, unlike laboratory criteria, which require equipment and instruments that might not be available in the hospital.

The strong association between severe maternal morbidity and admission to ICU, and between maternal near miss and oxygen saturation less than $90 \%$ over 1 hour, ventilation and intubation for a period of 1 hour or more not related to anesthesia, and cardiopulmonary resuscitation shows the severity of the clinical conditions of these women.

A study in Brazil by Amorim et al., ${ }^{19}$ who evaluated 1481 patients admitted to the obstetric ICU with criteria for near miss or severe morbidity, found that 291 were classified as near miss, with hypertension (78.4\%), hemorrhage (25.4\%), and infections (16.5\%) being the main causes of these admissions. Zanette et al. ${ }^{12}$ followed up 6706 women with severe hypertensive disorders and grouped the women with near miss and maternal death into one group, verifying that $74.2 \%$ (290/391) were admitted to the ICU, 71.4\% (279/391) used magnesium sulfate, $64.7 \%$ (253/391) were hospitalized for more than 7 days, and $29.7 \%(116 / 391)$ used mechanical ventilation.

The Magpie Trial Collaborative Group ${ }^{21}$ demonstrated that use of magnesium sulfate by women with severe pre-eclampsia can reduce the risk of eclampsia and subsequent maternal death, attesting to the need for its use in such situations. This was also demonstrated in the present study: use of magnesium sulfate was found to increase survival in severe maternal morbidity, representing a protective factor.

The present study has some limitations. First, the study outcome has a low prevalence, which resulted in wide confidence intervals. Second, the two study groups demonstrate clinical severity, which makes them similar to a certain extent. Third, neither the time taken before these women received health care nor the therapeutic route leading to their admission was evaluated; these factors are known to be related to poorer maternal and neonatal results. ${ }^{12-14,22-24}$ Additionally, data for newborns were missing from some medical records.

The study included only women with potentially life-threatening conditions after their admission to the institution. Therefore, it was not the objective to evaluate how the criteria of severe morbidity or near miss represent the risk of maternal death as compared with women without these characteristics. The main objective was to define within this population the criteria that are most strongly related to maternal death so that it will be possible to establish more effective care protocols with multidisciplinary teams trained for a rapid response when faced with these situations.

The study has strengths. First, its population is similar to that of numerous other studies, and the sample represents epidemiologically the obstetric population in economically disadvantaged regions, thereby allowing its findings to be extrapolated to other locations in Brazil and other countries. Second, the 1-year study period should take into account all possible seasonal interferences. Third, its approach was directed toward the criteria of near miss and severe maternal morbidity in an obstetric and neonatal referral center. The specific association of each one of these criteria was evaluated directly, in addition to how the interference between them can contribute to maternal death, identifying those that are principally responsible for the unfavorable outcome in this population. Accordingly, in a population classified as severe maternal morbidity or near miss, as defined by WHO, the main determining criteria for maternal death were eclampsia, low oxygen saturation, need for admission in ICU, need for intubation, mechanical ventilation, and cardiopulmonary resuscitation. The use of magnesium sulfate was found to be a protective factor.

\section{AUTHOR CONTRIBUTIONS}

The specific analytic approach used in the present study arose in a group discussion among all the authors. All authors developed the study protocol. The analyses were planned and performed by HMPL and FHCC. The first version of the manuscript was drafted by HMPL and GCN; all other authors, and particularly FHCC and FELF, contributed to revision.

\section{CONFLICT OF INTEREST}

The authors have no conflicts of interest.

\section{REFERENCES}

1. UN Millennium Campaign. About 2015 Millennium Campaign. UN Millennium Campaign, 2002. Published 2000. http://www.endpoverty2015.org/about/. Accessed January 19, 2015.

2. Hogan MC, Foreman KJ, Naghavi M, et al. Maternal mortality for 181 countries, 1980-2008: a systematic analysis of progress towards Millennium Development Goal 5. Lancet. 2010;375:1609-1623.

3. Ronsmans C. Graham WJ; Lancet Maternal Survival Series steering group. Maternal mortality: who, when, where, and why. Lancet. 2006;368:1189-1200. 
4. Brazil. DATASUS - SUS Department of Informatics. Health information. Vital Statistics. Deaths of women of childbearing age and maternal deaths - Brazil. Ministry of Health. Published 2015. http:// tabnet.datasus.gov.br/cgi/tabcgi.exe?sim/cnv/mat10uf.def. Accessed September 01, 2015.

5. Geller SE, Rosenberg D, Cox SM, et al. The continuum of maternal morbidity and mortality: factors associated with severity. Am J Obstet Gynecol. 2004;191:939-944.

6. Sousa MH, Cecatti JG, Hardy EE, Serruya SJ. Severe maternal morbidity (near miss) as a sentinel event of maternal death. An attempt to use routine data for surveillance. Reprod Health. 2008;5:6.

7. Say L, Souza JP. Pattinson RC; WHO working group on Maternal Mortality and Morbidity classifications. Maternal near miss-towards a standard tool for monitoring quality of maternal health care. Best Pract Res Clin Obstet Gynaecol. 2009;23:287-296.

8. Cecatti JG, Costa ML, Haddad SM, et al. Network for Surveillance of Severe Maternal Morbidity: a powerful national collaboration generating data on maternal health outcomes and care. BJOG. 2016;123:946-953.

9. Roopa PS, Verma S, Rai L, Kumar P, Pai MV, Shettyl J. "Near miss" obstetric events and maternal deaths in a tertiary care hospital: an audit. J Pregnancy. 2013;393758.

10. Troncon JK, De Quadros Netto DL, Rehder PM, Cecatti JG, Surita FG. Maternal mortality at a referral center of Southeastern Brazil. Rev Bras Ginecol Obstet. 2013;35:388-393.

11. Giordano JC, Parpinelli MA, Cecatti JG, et al. The burden of eclampsia: results from a multicenter study on surveillance of severe maternal morbidity in Brazil. PLoS ONE. 2014;9:e97401.

12. Zanette E, Parpinelli MA, Surita FG, et al. Maternal near miss and death among women with severe hypertensive disorders: a Brazilian multicenter surveillance study. Reprod Health. 2014; 11:4.

13. Rocha Filho EA, Costa ML, Cecatti JG, et al. Contribution of antepartum and intrapartum hemorrhage to the burden of maternal near miss and death in a national surveillance study. Acta Obstet Gynecol Scand. 2015;94:50-58.
14. Dias MA, Domingues RM, Schilithz AO, et al. Incidence of maternal near miss childbirth and postpartum hospital: survey data Born in Brazil. Cad Saúde Pública. 2014;30(Suppl.1):S169-S181.

15. Domingues RM, Dias MA, Nakamura-Pereira M, et al. Process of decision-making regarding the mode of birth in Brazil: from the initial preference of women to the final mode of birth. Cad Saúde Pública. 2014;30(Suppl.1):S101-S116.

16. Dias MA, Domingues RM, Pereira AP, et al. The decision of women for cesarean birth: a case study in two units of the supplementary health care system of the State of Rio de Janeiro [in Portuguese]. Ciênc Saúde Coletiva. 2008;123:64-67.

17. Chang J, Elam-Evans LD, Berg CJ, et al. Pregnancy-related mortality surveillance-United States, 1991-1999. MMWR Surveill Summ. 2003;52:1-8.

18. O'Connor HD, Hehir MP, Kent EM, et al. Eclampsia: trends in incidence and outcomes over 30 years. Am J Perinatol. 2013;30:661-664.

19. Amorim MMR, Katz L, Valença M, Araújo DE. Severe maternal morbidity in obstetric ICU in Recife, northeastern Brazil. Rev Assoc Med Bras. 2008;54:261-266.

20. WHO. WHO recommendations for the prevention and treatment of postpartum haemorrhage. WHO 2012. Published 2012. http://apps. who.int/iris/bitstream/10665/75411/1/9789241548502_eng.pdf. Accessed January 15, 2015.

21. Magpie Trial Collaborative Group. Do women with pre-eclampsia, and their babies, benefit from magnesium sulphate? The Magpie Trial: a randomised placebo-controlled trial. Lancet. 2002;359:1877-1890.

22. Amaral E, Souza JP, Surita F, et al. A population-based surveillance study on severe acute maternal morbidity (near-miss) and adverse perinatal outcomes in Campinas, Brazil: the Vigimoma Project. BMC Preg Childbirth. 2011;11:9.

23. Santana DS, Cecatti JG, Parpinelli MA, et al. Severe maternal morbidity due to abortion prospectively identified in a surveillance network in Brazil. Int J Gynecol Obstet. 2012;119:44-48.

24. Pacheco AJC, Katz L, Souza ASR, Amorim MMR. Factors associated with severe maternal morbidity and near miss in the São Francisco Valley, Brazil: a retrospective, cohort study. BMC Preg Childbirth. 2014;14:91. 